\title{
THE ASSESSMENT OF CURVED CENTERLINE GENERATION IN HDMAPS BASED ON POINT CLOUDS
}

\author{
J. C. Zeng ${ }^{1}$, K. W. Chiang ${ }^{1, *}$ \\ ${ }^{1}$ Dept. of Geomatics, National Cheng Kung University, No.1, Daxue Road, East District, Tainan, Taiwan - \\ (jessica31017tngs, kwchiang72)@gmail.com
}

KEY WORDS : point cloud, HD Maps, MLS, autonomous vehicle, road edge, lane line, centerline

\begin{abstract}
ABS TRACT:
Over the decades, autonomous driving technology has attracted a lot of attention and is under rapid development. However, it still suffers from inadequate accuracy in a certain area, such as the urban area, Global Navigation Satellite System (GNSS) hostile area, due to the multipath interference or Non-Line-of-Sight (NLOS) reception. In order to realize fully autonomous applications, High Definition Maps (HD Maps) become extra assisted information for autonomous vehicles to improve road safety in recent years. Compared with the conventional navigation maps, the accuracy requirement in HD Maps, which is $20 \mathrm{~cm}$ in the horizontal direction and $30 \mathrm{~cm}$ in 3D space, is considerably higher than the conventional one. Additionally, HD Maps consist of rich and high accurate road traffic information and road elements. For the requirement of high accuracy, conducting a Mobile Laser Scanning (MLS) system is an appropriate method to collect the geospatial data accurately and efficiently. Nowadays, digital vector maps are constructed by digitalizing manually on the collected data. However, the manual process spends a lot of manpower and is not efficient and practical for a large field. Therefore, this paper proposes to automatically construct the crucial road elements, such as road edge, lane line, and centerline, to generate the HD Maps based on point clouds collected by the MMS from the surveying company. The RMSEs in the horizontal direction of the road edge, lane line, and centerline are all lower than $30 \mathrm{~cm}$ in 3D space.
\end{abstract}

\section{INTRODUCTION}

Reacting to the era of autonomous driving, also called selfdriving, not only an industry but also academia has gotten involved in the relevant researches and development for autonomous vehicles. Autonomous vehicles dedicates to deliver better road safety to transport without human intervention and promise to bring several benefits including better fuel efficiency, less congestion and pollution, and reducing drivers' cost (Litman 2017). According to the definition of driving automation established by the Society of Automotive Engineers (SAE) International, the progress of self-driving development can be divided into six stages illustrated in Figure 1. Level 0 is no automation driving which means the driver has to carry out and concentrate on the whole driving process. Level 1 and Level 2 employ the functions of Advanced Driver Assistance System (ADAS), such as lane departure warning (LDWs), lane keeping assist systems (LKAS), and adaptive cruise control systems (ACC), to reduce the drivers' stress. However, the driver still needs to pay full attention to driving. For level 4, the automated driving system (ADS) can handle all driving tasks by itself instead of certain situations (NHTSA).

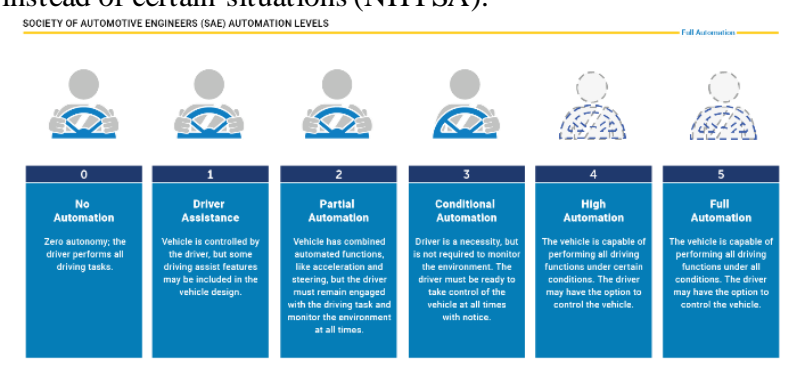

Figure 1. SAE automation levels (reference: NHTSA)
Generally, there are many sensors configured on the autonomous vehicle to support the navigation and perception services. These sensor sy stems can be separated into positioning sensors, such as Global Navigation Satellite Sy stem (GNSS), Inertial Navigation System(INS), and wheel-mounted Distance Measuring Indicator (DMI), and mapping sensors, such as camera, LiDAR, radar. Although the Positioning and Orientation System (POS), which integrates GNSS and INS, has been fully developed and widely used in our daily life, the GNSS signals are easily disturbed by multipath interference and Non-Line-Of-Sight (NLOS) reception in the urban canyon. It might lead to considerable positioning errors and unexpected accidents. Additionally, the mapping sensors are also inferior to inherent limitation, for example, camera is affected by the illumination, LiDAR suffers from the cost, and radar is limited to the spatial resolution. To overcome this dilemma, HD Maps becomes extra assisted information for autonomous vehicle. Compared with the conventional digital maps, HD Maps consist of rich road information, such as road geometry, road edge, lane line, centerline, traffic sign, traffic light, with highly precise accuracy. Moreover, all road elements in HD Maps defined in 3D space with equal scales of the real world to prevent misinterpretation. As well as, dynamic information is also recorded and shared in real-time (Harsha, 2017).

Concerning the accuracy requirement for various applications, the accuracy can be divided into four classes: Which Road, Which Lane, Where In Lane, and Active Control. Among them, the total error budget from positioning and mapping need to be lower than 1.5 meters for Which Lane application (Stephenson et al. 2011). Otherwise, the accuracy requirement in HD Maps should reach 0.2 meters in the horizontal direction and 0.3 meters in 3D space. In order to satisfy the requirements and characteristics of HD Maps, Mobile Laser Scanning (MLS) system, one branch of Mobile Mapping System (MMS), is a proper method to collect geospatial information with high

\footnotetext{
* Corresponding author
} 
accuracy rapidly and efficiently. For the typical mapping task digitalization is usually conducted manually which is timeconsuming and wasting manpower. Therefore, this study proposes to develop an automated feature extraction method based on point cloud data acquired by the MLS system, which takes advantage of extracting high-density 3D points representing the surface of objects and high resolution of 3D topographic data (Kukko, 2013). On the other hand, this study takes the modelling process into consideration to fit the extracted road elements since the HD Maps should be light vector maps rather than extracted point cloud for uploading and downloading efficiency (Gwon et al., 2016). Finally, generating the centreline and evaluating the results with verified HD Maps to guarantee the quality.

\section{RELATED WORK}

\subsection{Road Surface Extraction}

Since this study only focuses on the features on the road surface, removing non-ground point clouds or useless points are contributed to subsequent extraction processing. Therefore, the first step is to obtain the correct road surface to improve the extracted results and computational efficiency. The researches for road surface extraction can be simply categorized into 2D image-based methods and 3D point cloud processing respectively For 2D image-based method, there are many studies performed deep learning algorithms to segment the road surface from the background. Rezaee and Zhang (2017) conducted a patchedbased Deep Neural Network (DNN) model to detect roads on high spatial resolution orthophoto aerial data. Dai et al. (2017) applied the K-mean algorithm to divide the objects into road, sky, and background as well as using the Convolution Neural Network $(\mathrm{CNN})$ to classify different road types. Instead of implementing deep learning methods and processing the images directly, Yang et al. (2012) generated the georeferenced image from laser points to represent the geospatial information of surroundings. The road surface can be determined by grey-value and elevation filtering.

In contrast to 2D image-based methodologies, processing the 3D point cloud directly can avoid accuracy and data loss and maintain the whole process on a global scale. Smadja et al. (2010) performed RANdom SAmple Consensus (RANSAC) algorithm on each scan line of point cloud individually to extract the road surface. On the other hand, Hervieu and Soheilian (2013) proposed to detect the curb by computing the normal vector of the point cloud because the curbs are almost vertical to the road surface. Afterward, the point clouds between these two curbs are regarded as the road surface. Similarly, Ma (2017) and Guan et al. (2014) partitioned the point cloud into a series of profiles along with the MLS trajectory data. These profiles are further cut into many grids to extract the curb points based on the conditions of elevation difference and slope changing.

\subsection{Road Markings Extraction}

Road markings are vital road elements for guidance, warning, and prohibition for all road users. On the other hand, road markings are supported for the operation of autonomous vehicles and reliable centerline generation. In general, the road markings are high retro-reflective pattern on the road surface. It is a critical feature for road marking distinguishing. The road markings extraction researches can be categorized into carrying out 2D images converted from MLS points and MLS point clouds directly.

For the image-based method, Wen et al. (2019) generated the 2D image whose grey values are calculated from the mean value of intensity of the points in each grid. Then, segmenting the road markings from the background based on the modified segmentation network, U-net. To determine the semantic information of the road markings, Euclidean Clustering (EC) algorithm and the CNN model are used for road markings classification. Additionally, Yu et al. (2014) proposed to filter out the non-road markings by Otsu's algorithm, which is an adaptive thresholding method, since the intensity value is vary depending on the distance between the scanner center and measured object and incident angles of the emitted laser beams. These extracted road markings are further classified by adopting Deep Boltzmann Machines (DBM) and further using Principal Component Analy sis (PCA).

Except for using deep learning methods, Yang et al. (2012) applied Progressive Probabilistic Hough Transformation (PPHT) operator to extract the linear road marking extraction. Accordingly, Cheng et al. (2016) extracted the road markings from the enhanced 2D intensity images and clustered the road markings by neighbor-counting filtering and region growing algorithm. Furthermore, classifying these road markings based on the minimum bounding rectangle (MBR) algorithm and decision tree.

\subsection{Centerline Generation and Modelling}

Centerline is an essential road element for autonomous vehicle control systems. Cudrano et al. (2020) proved that the estimated centerline information is contributed to promoting the vehicular control system. From the literature review, there are many pieces of research for centreline generation based on various data recourse. The accuracy of the centerline generated by the GNSS trajectory is relevant to the GNSS quality. As well as, it is impractical for the large test field due to the length and number of lanes on the road (Gwon et al., 2016). Additionally, Zhao (2017) partitioned the road markings into several blocks along the trajectory. Then, the centreline can be created by calculating the center point in each block based on the midpoint formula from the bilateral lane line points. On the other hand, Guan (2013) and $\mathrm{Ma}(2017)$ proposed to generate the centerline parallel to the horizontal curved lane line extracted from the MLS points for ensuring road safety.

Storage efficiency and data usability are also important for HD Maps. The extracted road edges or road markings points cannot become the road elements stored in the HD Maps due to a large amount of data volume. Therefore, the modelling process is required to represent the geometry information, reach maps assess efficiency, and satisfied with the specific HD Maps format standard. The clothoid algorithm is the best fitting model for depicting the road geometry and the smooth transition curve since the road is traditionally constructed corresponding to the clothoid (Marzbani et al. 2015). However, the clothoid is only defined in the 2D space. On the other hand, Xu et al. (2009) employed the B-spline model to fit the centreline generated by two boundary lines since B-spline is flexible to adjust the location of control points without changing the entire shape of the curve. Therefore, B-spline is widely developed in computer-aided design (CAD). Rather than giving control points and pre-set information, Guan (2013) and $\mathrm{Ma}$ (2017) interpolating a smooth curve based on a cubic spline algorithm, which is consisted of sequential three-degree polynomial functions.

\section{METHODOLOGY}

The methodology in this study can be separated into feature extraction and road elements modelling illustrated in Figure 2 and Figure 3. 


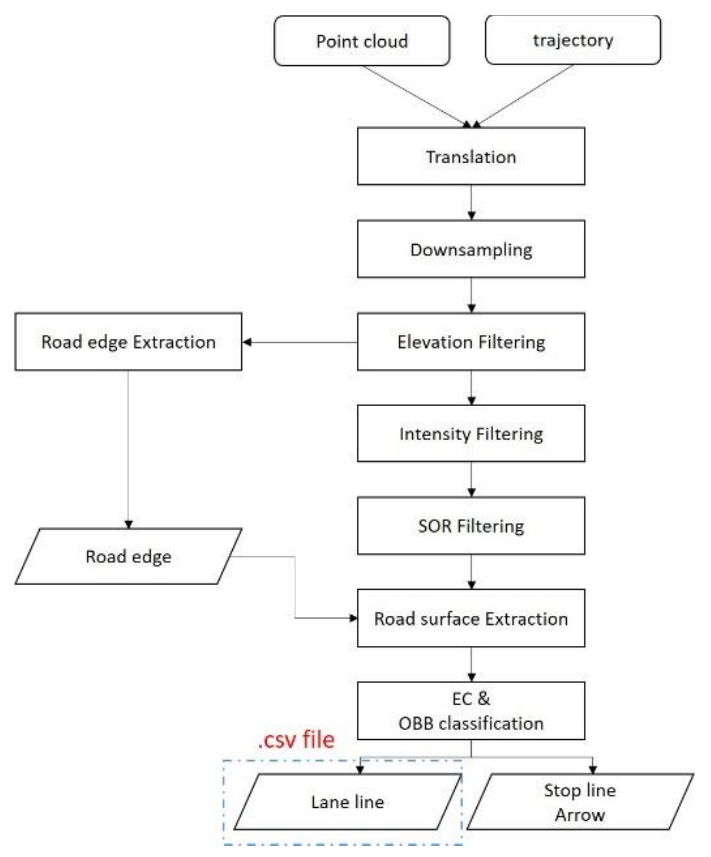

Figure 2. The flowchart for feature extraction

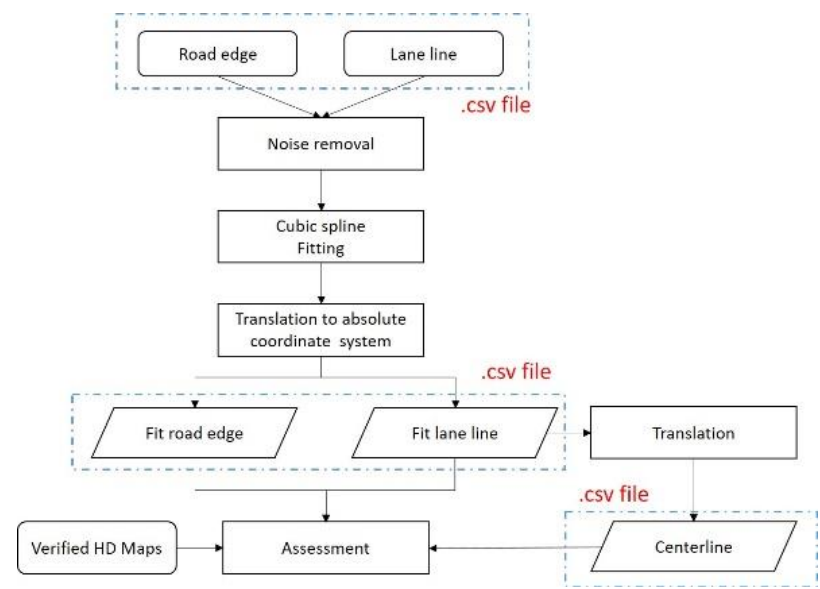

Figure 3. The flowchart for road elements modelling

\subsection{Road surface extraction}

The point clouds collected by the MLS system provided by the surveying company is a large amount of data volume. So as to carry out these point clouds, point cloud pre-processing is an essential step before extracting the road surface to reduce the computational time and improve the quality of the results. First, the MLS point clouds, defined in an absolute coordinate system, are translated to the local level frame to decline the computing costs. Meanwhile, the point clouds are downsampled by using the Voxel Grid filter to approximate the points in every segmented voxel with the representative point under each voxel. It considerably drops down the number of point clouds without losing the features of the targets. Subsequently, since the distance between the IMU, representing the position of MLS trajectory data, and the road surface is ideally constant, the ground point clouds can be determined by setting the buffered range from the trajectory data. The non-ground point clouds, such as trees, traffic light, and traffic signs, are roughly eliminated. In the next step, the ground point clouds are partitioned into a set of profiles and voxelized into numerous voxels with a given size. The study areas lack curb structure, so the candidate curb points are defined by the intensity difference. Because the intensity of the sidewalk beside the road is significantly high, this characteristic is seemed to be a key factor to extract the road edge. If the intensity difference of two centroids in adjacent voxels is higher than the given threshold, the point will be considered to be the curb point. However, there existed wrong curb points derived from the road markings points, so these points are removed to refine the curb points.

\subsection{Road Markings extraction}

The essential element for centreline generation is lane line, which is linear road marking, due to its geometrical characteristics. On the other hand, the road markings high retro-reflective paintings on the asphalt road, so the intensity of road markings is far higher than the intensity of asphalt road. These road markings are easily distinguished by implementing intensity filtering on the ground point clouds with a given threshold. Then, the Statistic Outlier Removal (SOR) filter is used to filter out the noisy points rested from the intensity filtering. Instead of extracting the whole road surface, the refined curbs points are employed to capture the road marking points on the road surface from the point clouds processed by intensity filtering and SOR filter. Since the point clouds are unorganized, the next step is to cluster the road marking points by Euclidean Clustering (EC) algorithm based on the distance threshold. If the distance between two arbitrary points is larger than the threshold, these two points are differentiated into different clusters. Otherwise, these two points are in the same cluster. However, the EC method is unable to segment the stop line and lane line since they are connected with each other. This study proposes to segment the road marking points into several blocks along the trajectory. If the point density in each block, which calculated by the number of point clouds divides the area of block, is higher than the density threshold, the block is viewed as the location of the stop line. After separating the connected road markings, the Oriented Bounding Box (OBB) algorithm is applied to capture the width and length of each cluster. OBB method can derive the major orientation of the object based on PCA to construct a bounding box wrapped in each cluster. Therefore, these clusters can be classified into different types of road markings based on the geometry information defined in the road markings design standards.

\subsection{Road Elements Modelling and Centerline Generation}

The classified road marking points are not proper for use as road map due to large storage space. In addition, it is hard to elucidate the road geometry information (Gwon et al., 2016). This study conducts the cubic spline fitting algorithm, which is widely used in many pieces of research and proved to perform outstandingly for road geometry fitting (Gwon et al., 2016; Ma, 2017), to model the extracted road edges and lane lines. The cubic spline curve is comprised of a series of continuous piecewise cubic polynomial functions between two sequential breakpoints to describe the smoothing in every interval. The cubic spline interpolation function is expressed as follows.

$F_{i}=\left\{\begin{array}{c}\sum_{i=1}^{n}\left[a_{i}+b_{i}\left(x-x_{i}\right)+c_{i}\left(x-x_{i}\right)^{2}+d_{i}\left(x-x_{i}\right)^{3}\right], \text { where } x_{i} \leq x \leq x_{i+1} \\ \sum_{i=1}^{n}\left[e_{i}+f_{i}\left(y-y_{i}\right)+g_{i}\left(y-y_{i}\right)^{2}+h_{i}\left(y-y_{i}\right)^{3}\right], \text { where } y_{i} \leq y \leq y_{i+1} \\ \quad(i=1,2, \ldots, n-1)\end{array}\right.$

where $\quad a_{i} \sim h_{i}=$ parameters for cubic spline functions

$x, y=$ the coordinate of point clouds

$x_{i}, y_{i}=$ the coordinate of break points

$\mathrm{n}=$ total numbers of interval 
The advantage of the cubic spline algorithm is that it uses low degree polynomial functions to fulfill the interpolation in small intervals to avoid the vibration instability due to high degree fitting polynomials and reduce the computational complexity for a large amount of data. However, the cubic spline is susceptible to the noisy points, so the noise removal is a necessary stage before modelling.

Owing to obey the road construction design, the centerline should be parallel to the lane line for traffic safety. Since the road width is fixed ideally, the centreline can be created by translating a certain distance from the central fitted lane line. Concerning the HD Maps format standard defined in Autoware, which is notable and widely used platform for autonomous driving development, the fitting results are saved as CSV format containing 3D absolute coordinates. Finally, the quality and accuracy of the results are assessed with the verified HD Maps to ensure the data quality and evaluate the availability of the proposed method.

\section{RESULT AND ANALYSIS}

\subsection{Study Area and Data Acquisition}

The study area is located in the Taiwan CAR Lab, which is the first domestic autonomous vehicle test proving ground. Taiwan CAR Lab is designed for simulating domestic traffic scenarios including intersection, roundabout, merging lane, and curved road shown in Figure 4. The lengths of the experimental areas are about 35 meters and 37 meters with four-lane road sections demonstrated in Figure 5.

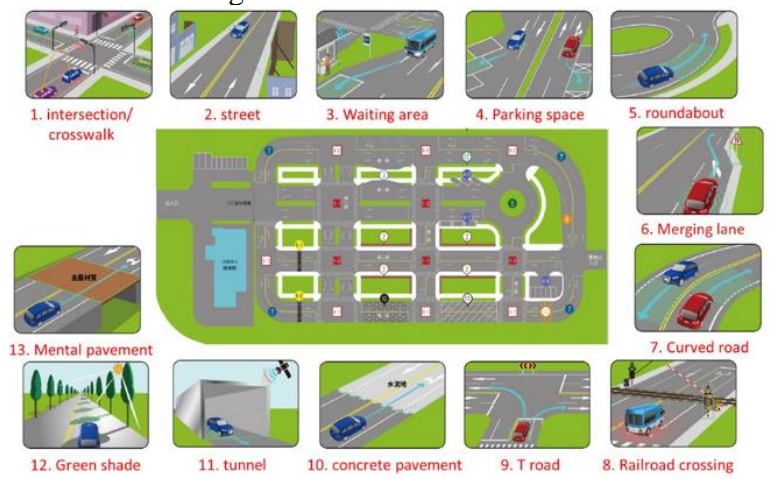

Figure 4. The designing schematic map of Taiwan CAR Lab

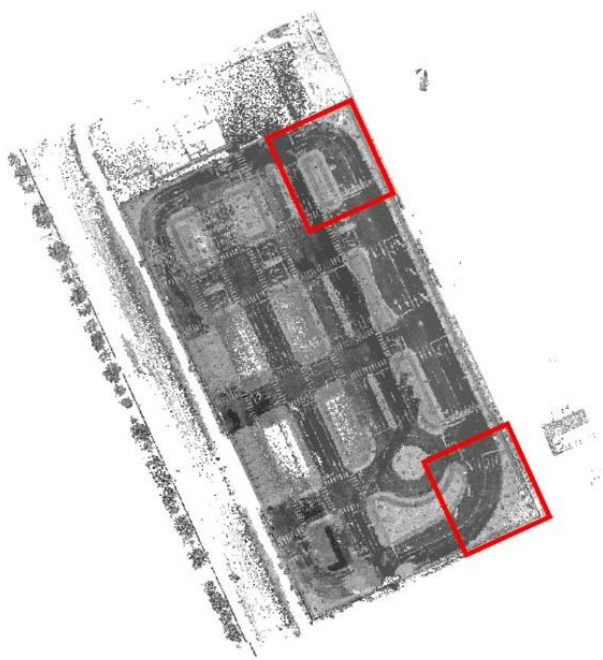

Figure 5. The designing schematic map of Taiwan CAR Lab
The point clouds and trajectory data are collected by the MLS system, RIEGL VMX-250, provided by the professional surveying company. RIEGL VMX-250 sy stem is composed of a POS system and sensing sensors. Among these mounted sensors, the POS system composed of dual-constellation GNSS and INS integrated system to maintain the stability of 0.005 degrees and 0.008 degree gyro drift for roll\&pitch and heading respectively and DMI, while the mapping sensors contains laser scanning, which can reach 6000,000 measurements per second, and industrial-grade digital cameras to provide high-resolution images.

\subsection{Results and Discussion}

In order to evaluate the accuracy of the results, the existed verified HD Maps for Taiwan CAR Lab are considered to be the ground truth for assessment. The ArcGIS 10.5 Desktop is implemented to display and analyze the performance of the proposed methodology by overlapping the modelling results and the verified HD Maps. Figure 6 and Figure 7 show the two curve scenarios overlapping with the results and verified HD Maps in this study. Since the ArcGIS only supports 2D demonstration, the verified HD Maps are converted to CAD files and divided into one point per centimeter to represent the ground truth data for accuracy assessment.

In addition, this study selects all points in each road element and calculates the statistical data. For the first scenario, the RMSEs in 3D space of road edge, lane line, and centreline are $0.210 \mathrm{~m}$, $0.083 \mathrm{~m}$, and $0.204 \mathrm{~m}$, respectively. While the RMSEs in 3D space of road edge, lane line, and centerline are $0.143 \mathrm{~m}, 0.049$ $\mathrm{m}$, and $0.078 \mathrm{~m}$ for the second scenario. The details of accuracy are illustrated in Table 1. From the results, the road edge modelling is more difficult than other road elements due to its complexity and uncertainty for determining the location of the boundary, especially in the scenario without curbs. Therefore, there are some twisted points located on the road edges in the first scenario shown in Figure 6. Moreover, the modelling road edge might contain a few points derived from the lane line points near the real road edge since the road edges are determined by the intensity difference. It leads to the error budget from the distance between the lane line and road edge and results in poor modelling results for road edges displayed in Figure 7. Therefore, the accuracy of the road edge is worse than the accuracy of lane lines, which are modelled from the clear and correct extracted lane line points. On the other hand, since the centerlines are generated from the central lane line, which is a double yellow line in these experimental areas, the error in modelling central lane line is propagated to the generated centerline. Furthermore, the elevation is also a source of error since the elevation of centerline cannot be considered by the translating method. Therefore, the accuracy of the centerlines cannot be as good as the accuracy of the lane lines.

Overall, the accuracy of all modelling road elements is lower than $30 \mathrm{~cm}$ in $3 \mathrm{D}$ space, which is satisfied with the accuracy requirement of HD Maps in 3D space. 


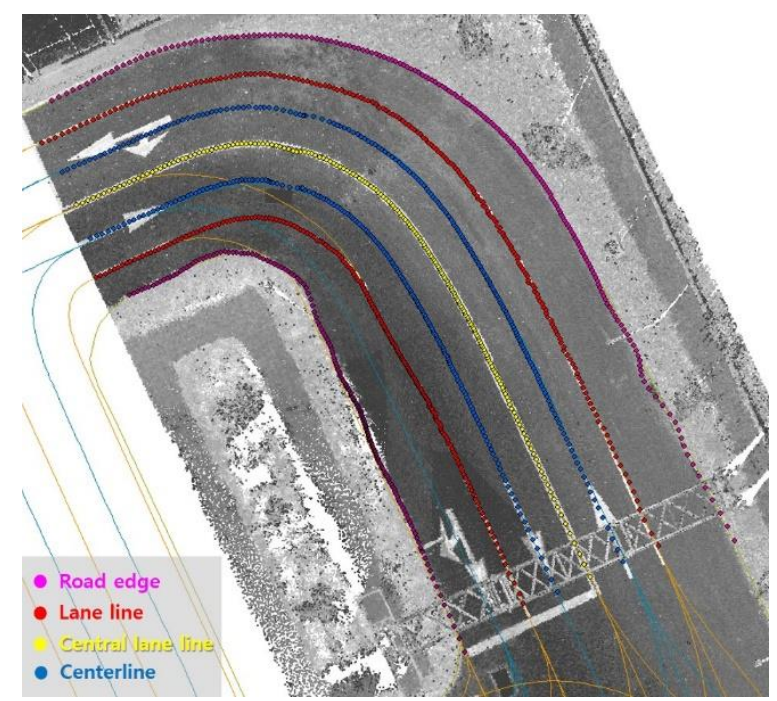

Figure 6. Modelling road elements in first scenario overlapping with verified HD Maps (pink points represent the road edge; red points represent the lane line; yellow points represent the central lane line; blue points represent the centerline)

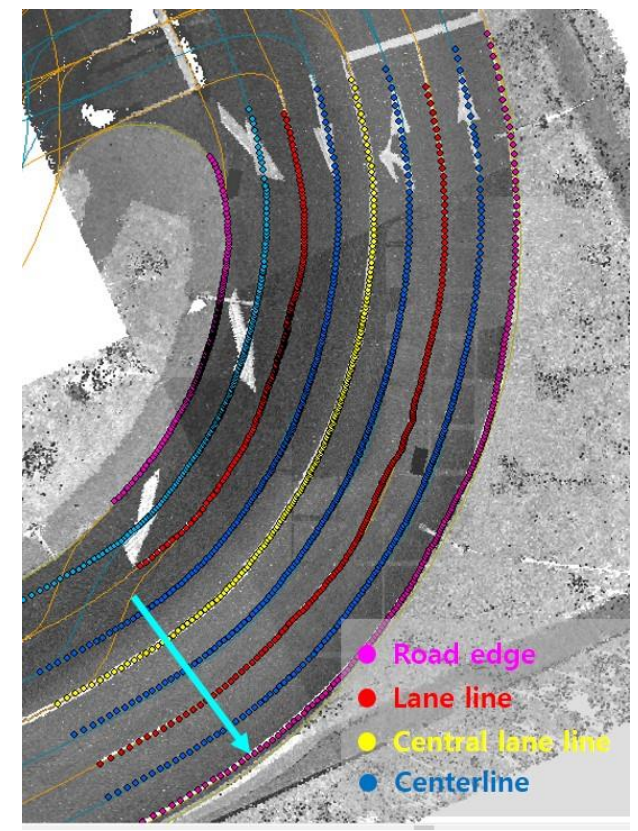

Figure 7. Modelling road elements in second scenario overlapping with verified HD Maps (pink points represent the road edge; red points represent the lane line; yellow points represent the central lane line; blue points represent the centerline)

\begin{tabular}{|l|c|c|c|}
\hline \multicolumn{5}{|c|}{ Scenario 1} \\
\hline & Road edge & Lane line & Centerline \\
\hline 2D Mean $(\mathrm{m})$ & 0.101 & 0.033 & 0.056 \\
\hline 3D Mean $(\mathrm{m})$ & 0.115 & 0.044 & 0.065 \\
\hline 2D STD $(\mathrm{m})$ & 0.093 & 0.026 & 0.047 \\
\hline 3D STD $(\mathrm{m})$ & 0.084 & 0.022 & 0.042 \\
\hline 2D RMSE $(\mathrm{m})$ & 0.137 & 0.042 & 0.073 \\
\hline 3D RMSE $(\mathrm{m})$ & 0.143 & 0.049 & 0.078 \\
\hline 2D Max $(\mathrm{m})$ & 0.385 & 0.115 & 0.181 \\
\hline 3D Max $(\mathrm{m})$ & 0.386 & 0.120 & 0.182 \\
\hline
\end{tabular}

\begin{tabular}{|l|c|c|c|}
\hline $\begin{array}{l}\text { Number of } \\
\text { points }\end{array}$ & 376 & 566 & 380 \\
\hline \multicolumn{4}{|c|}{ Scenario 2 } \\
\hline & Road edge & Lane line & Centerline \\
\hline 2D Mean $(\mathrm{m})$ & 0.147 & 0.060 & 0.084 \\
\hline 3D Mean $(\mathrm{m})$ & 0.151 & 0.070 & 0.190 \\
\hline 2D STD $(\mathrm{m})$ & 0.148 & 0.049 & 0.057 \\
\hline 3D STD $(\mathrm{m})$ & 0.146 & 0.045 & 0.073 \\
\hline 2D RMSE $(\mathrm{m})$ & 0.209 & 0.077 & 0.101 \\
\hline 3D RMSE(m) & 0.210 & 0.083 & 0.204 \\
\hline 2D Max $(\mathrm{m})$ & 0.547 & 0.221 & 0.210 \\
\hline 3D Max $(\mathrm{m})$ & 0.548 & 0.222 & 0.309 \\
\hline $\begin{array}{l}\text { Number of } \\
\text { points }\end{array}$ & 371 & 547 & 736 \\
\hline
\end{tabular}

Table 1 . The accuracy of modelling road elements

\section{CONCLUSIONS}

This study proposes to automatically generate the centreline on the curved road section based on MLS point clouds and model the certain road markings to build the light HD Maps to achieve better usability and storage efficiency for autonomous vehicles. Additionally, the accuracy of the modelling results is verified with the verified HD Maps to guarantee the data quality. The RMSEs of the modelling results are almost lower than $20 \mathrm{~cm}$ in the horizontal direction and $30 \mathrm{~cm}$ in $3 \mathrm{D}$ space, which reaches the accuracy requirement of HD Maps.

\section{ACKNOWLEDGEMENTS}

The authors would acknowledge the supports provided by the Ministry of the Interior.

\section{REFERENCES}

Cheng, M., Zhang, H., Wang, C., \& Li, J., 2016. Extraction and classification of road markings using mobile laser scanning point clouds. IEEE Journal of Selected Topics in Applied Earth Observations and Remote Sensing, 10(3), 1182-1196.

Cudrano, P., Mentasti, S., Matteucci, M., Bersani, M., Arrigoni, S., \& Cheli, F., 2020. Advances in centerline estimation for autonomous lateral control. arXiv preprint arXiv:2002.12685.

Dai, J. M., Liu, T. A. J., \& Lin, H. Y., 2017. Road surface detection and recognition for route recommendation. 2017 IEEE Intelligent Vehicles Symposium (IV), 121-126.

Guan, H., Li, J., Yu, Y., Wang, C., Chapman, M., \& Yang, B., 2014. Using mobile laser scanning data for automated extraction of road markings. ISPRS Journal of Photogrammetry and Remote Sensing, 87, 93-107.

Gwon, G. P., Hur, W. S., Kim, S. W., \& Seo, S. W., 2016. Generation of a precise and efficient lane-level road map for intelligent vehicle systems. IEEE Transactions on Vehicular Technology, 66(6), 4517-4533.

Hervieu, A., \& Soheilian, B., 2013. Semi-automatic road/pavement modeling using mobile laser scanning. ISPRS Annals of the Photogrammetry, Remote Sensing and Spatial Information Sciences, 2, 31-36.

Harsha V., 2017. HD maps: New age maps powering autonomous vehicles. Geospaial world. 
Kukko, A., 2013. Mobile Laser Scanning-Sy stem development, performance and applications. Finnish Geodetic Institute.

Litman, T., 2018. Autonomous vehicle implementation predictions. Victoria Transport Policy Institute, Victoria, Canada.

Marzbani, H., Jazar, R. N., \& Fard, M., 2015. Better road design using clothoids. Sustainable automotive technologies 2014. 2540.

Ma, L., 2017. Generation of Horizontally Curved Driving Lines for Autonomous Vehicles Using Mobile Laser Scanning Data. MSthesis. University of Waterloo.

National Highway Traffic Safety Administration. https://www.nhtsa.gov/technology -innovation/automated-

vehicles-safety

Rezaee, M., \&Zhang, Y., 2017. Road detection using deep neural network in high spatial resolution images. 2017 Joint Urban Remote Sensing Event, 1-4.

Smadja, L., Ninot, J., \& Gavrilovic, T., 2010. Road extraction and environment interpretation from LiDAR sensors. IAPRS, 38(281-286), 1 .

Stephenson, S., Meng, X., Moore, T., Baxendale, A. and Ford, T., 2011. Accuracy requirements and benchmarking position solutions for intelligent transportation location based services. The 8th International Symposium on Location-Based Services.

Wen, C., Sun, X., Li, J., Wang, C., Guo, Y., \& Habib, A., 2019. A deep learning framework for road marking extraction, classification and completion from mobile laser scanning point clouds. ISPRS journal of photogrammetry and remote sensing, 147, 178-192.

Xu, H., Wang, X., Huang, H., Wu, K., \& Fang, Q., 2009. A fast and stable lane detection method based on B-spline curve. 2009 IEEE 10th International Conference on Computer-Aided Industrial Design \& Conceptual Design, 1036-1040.

Yang, B., Fang, L., Li, Q., \& Li, J., 2012. Automated extraction of road markings from mobile LiDAR point clouds. Photogrammetric Engineering \& Remote Sensing, 78(4), 331338.

Yu, Y., Li, J., Guan, H., Jia, F., \& Wang, C., 2014. Learning hierarchical features for automated extraction of road markings from 3-D mobile LiDAR point clouds. IEEE Journal of Selected Topics in Applied Earth Observations and Remote Sensing, 8(2), 709-726.

Zhao, H., 2017. Recognizing features in mobile laser scanning point clouds towards 3D high-definition road maps for autonomous vehicles. MS thesis. University of Waterloo. 